\title{
Quantum electromagnetic phenomena far from small evaporating black holes
}

\author{
Slava Emelyanov \\ Institute of Theoretical Physics, Karlsruhe Institute of Technology (KIT), \\ 76128 Karlsruhe, Germany \\ email: viacheslav.emelyanov@kit.edu
}

\begin{abstract}
One might expect far away from physical black holes that quantum field quantisation performed in Minkowski space is a good approximation. Indeed, all experimental tests in particle colliders reveal no deviations so far. Nevertheless, the black holes should leave certain imprints of their presence in quantum processes. In this paper, we shall discuss several local imprints of small, primordial evaporating black holes in quantum electrodynamics in the weak gravity regime. Physically this can be interpreted as being macroscopic manifestations of vacuum fluctuations.
\end{abstract}

Keywords. Black hole physics, gravitation, elementary particles

\section{Introduction}

In quantum field theory observables are self-adjoint, local operators. These local operators could be of various nature. In what follows we consider observables corresponding to the electromagnetic field. Specifically, these could be an electromagnetic wave propagating from an emitter to a receiver and an electrostatic field sourced by a point-like charge, both being studied in a box of about one-cubic-meter size.

It is a consequence of the requirements needed to formulate a physically acceptable quantum field theory (e.g., Haag 1996) that quantum operators localised in a certain space-time region probe the vacuum as if it is a non-empty state. The physical interpretation of this effect can be readily given in terms of the quantum or vacuum fluctuations. These fluctuations or quantum noise can show up at microscopic as well as macroscopic scales. The formation of inhomogeneities like galaxies and clusters of galaxies in our universe is their most remarkable macroscopic manifestation (Mukhanov \& Chibisov 1981).

According to the Tomita-Takesaki theorem (Haag 1996), the quantum vacuum satisfies a Kubo-Martin-Schwinger aka thermal condition with respect to any localised set of the field operators and a certain one-parametric group of its automorphism. This group could be of a geometrical as well as non-geometric origin, i.e. generated by a Killing or nonKilling vector, respectively. The lesson of this is that the quantum vacuum can respond as a many-particle state which is mainly a property of quantum field operators, rather than of the vacuum state.

\section{Quantum vacuum as plasma-like medium}

Vacuum as dielectric. The quantum vacuum possesses a dielectric-like property in the absence of any external field with one of its observable/measurable manifestation being a part of the Lamb shift. The "dipoles" of the vacuum are virtual $e^{+} e^{-}$pairs. This effect follows from taking into account the one-loop correction to the photon self-energy which leads to the running electric charge (e.g., Peskin \& Schroeder 1995).

Specifically, the full photon propagator in quantum field theory is diagrammatically 
given by

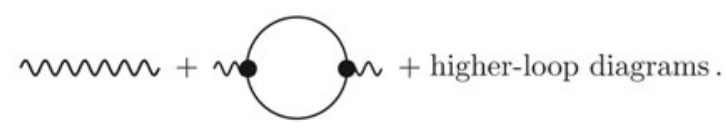

The loop diagrams lead to the modification of the classical field equation. This becomes in momentum space

$$
\left(k^{2} \eta^{\mu \nu}-k^{\mu} k^{\nu}-\Pi^{\mu \nu}(k)\right) a_{\nu}(k)=0,
$$

where $\Pi^{\mu \nu}(k)$ is a polarisation tensor vanishing in the limit of the vanishing fine structure constant $\alpha$. The gauge symmetry entails $\Pi^{\mu \nu}(k)=\left(k^{2} \eta^{\mu \nu}-k^{\mu} k^{\nu}\right) \Pi\left(k^{2}\right)$. Thus, one obtains $k^{2}\left(1-\Pi\left(k^{2}\right)\right) a^{\mu}=0$ for the photon $\left(k^{\mu} a_{\mu}=0\right)$. The extra factor $1-\Pi\left(k^{2}\right)$ leads to the charge renormalisation and, hence, to the modification of the classical Coulomb interaction at scales of the order of the Compton length of the electron. The resulting effect is the screening of the charge by the virtual $e^{+} e^{-}$pairs like in a dielectric.

Vacuum as magnetised plasma. Probing the quantum vacuum by studying how an electromagnetic wave propagates through a spacetime region with a super-strong magnetic field $\left(B \gg(\pi / \alpha) B_{c}\right.$, where $\left.B_{c}=m_{e}^{2} / e\right)$, the vacuum shows up a property inherent to a magnetised plasma, i.e. the plasma held at a static external magnetic field (Melrose \& Stoneham 1976 and Dittrich \& Gies 2000).

Due to a collective response of the plasma particles being tied to the external magnetic field lines, the propagation of an electromagnetic wave is significantly dependent on its polarisation (e.g., Bittencourt 2004). Specifically, an electromagnetic wave polarised in the direction coplanar to the plane defined by its spreading direction $\mathbf{k}$ and the external magnetic field $\mathbf{B}$ propagates along the magnetic field lines. This is known as the Alfvén wave.

The external magnetic field $B$ in the vacuum entails the modification of the vacuum polarisation tensor $\Pi^{\mu \nu}(k, B)$ which becomes non-diagonal. This is due to $B \neq 0$ which enters the field equations and, hence, modifies the electron and photon propagator. At one-loop approximation it turns out that a light wave polarised in the direction perpendicular to the plane defined by $\mathbf{k}$ and $\mathbf{B}$ is oblivious to the external magnetic field, i.e. $\omega^{2}=|\mathbf{k}|^{2}$. This resembles a behaviour of the fast magneto-acoustic wave in case of the Alfvén velocity $v_{A}=|\mathbf{B}| / \sqrt{4 \pi \rho} \gg 1$, where $\rho$ is a plasma-like density of the vacuum. If the polarisation vector $a_{\mu}$ is coplanar to that plane, then, as it was found by Melrose \& Stoneham (1976), $\omega^{2}=|\mathbf{k}|^{2} \cos ^{2} \theta$, where $\theta$ is an angle between $\mathbf{k}$ and $\mathbf{B}$. This corresponds to the Alfvén wave in case the condition $v_{A} \gg 1$ is fulfilled.

Vacuum as neutral plasma. The quantum vacuum responds non-trivially in the background of evaporating black holes, namely it acquires plasma-like properties (Emelyanov 2016a).

To demonstrate the effect, one needs to compute the vacuum polarisation tensor. It must depend a priori on a mass $M$ of the hole and a distance $R$ to its centre. The former dependence arises since when $M=0$ the problem reduces to that in flat space-time. The latter one is, however, due to our expectation that local physics in the asymptotically flat region $(R \rightarrow \infty)$ should reduce to the familiar Minkowskian one.

A part of the tensor $\Pi^{\mu \nu}(k, M, R)$ was computed at one-loop level by Drummond \& Hathrell (1980). However, it gets an extra term due to the quantum effect entailing the black-hole evaporation. Specifically for the semi-classical theory be reliable, a field operator in the background of any black hole should be expanded over modes in which the vacuum expectation value of stress tensor does not diverge on the (future) horizon. 
This yields the electron propagator in momentum space of the following form

$$
S\left(k, k^{\prime}\right) \approx\left(\not k+m_{e}\right)\left(\frac{i}{k^{2}-m_{e}^{2}+i \varepsilon}-2 \pi\left(\frac{27 r_{H}^{2}}{16 R^{2}}\right) \frac{\delta\left(k^{2}-m_{e}^{2}\right)}{e^{k_{0} / T_{H}}+1}\right) \delta\left(k-k^{\prime}\right),
$$

where $T_{H}=M_{\mathrm{Pl}}^{2} / 8 \pi M$ is Hawking's parameter, $M_{\mathrm{Pl}}=(\hbar / G)^{\frac{1}{2}}$ the Planck mass, $r_{H} \equiv$ $2 M G$ a size of the black-hole horizon. We have omitted in (2.3) terms of sub-leading order vanishing as $1 / R^{3}$ at the spatial infinity. The propagator (2.3) is valid only for $\left|r-r^{\prime}\right| \ll R$ and $r_{H} \ll R$ (Emelyanov 2016a \& 2016b). We should mention that it is legitimate in this regime to employ flat coordinates as space-time is locally Minkowski.

The geometrical part of $\Pi^{\mu \nu}(k, M, R)$ drops out at least as fast as $1 / R^{3}$ at spatial infinity, whereas its vacuum part vanishes as $1 / R^{2}$ as can be seen in (2.3). Therefore, the main contribution to $\Pi^{\mu \nu}(k, M, R)$ is due to the second term in (2.3).

The modification of the electron propagator has a thermal-like structure. In case of a large black hole $\left(m_{e} \gg T_{H}\right)$ the one-loop self-energy of the photon is exponentially suppressed by the Boltzmann factor $\exp \left(-m_{e} / T_{H}\right)$. Therefore, we consider a small black hole with $T_{H} \gg m_{e}$ in the following. This corresponds to the black-hole mass $M \ll 10^{16} \mathrm{~g}$ and, thus, justifies the hard thermal loop approximation (e.g., Le Bellac 1996) in which one can omit the electron mass $m_{e}$ as being negligible with respect to the temperature parameter $T_{H}$.

At one-loop level the photon propagator acquires two poles. One of these corresponds to a transverse mode, the photon, with $\omega^{2}=|\mathbf{k}|^{2}+\pi_{T}(\omega,|\mathbf{k}|)$, another one to a longitudinal mode with $\omega^{2}=|\mathbf{k}|^{2}+\pi_{L}(\omega,|\mathbf{k}|)$ which is known as a plasmon. The functions $\pi_{T}(\omega,|\mathbf{k}|)$ and $\pi_{L}(\omega,|\mathbf{k}|)$ are the same as found by Weldon (1982), but with the temperature

$$
T_{L} \approx \frac{3 \sqrt{3}}{16 \pi} M_{\mathrm{Pl}} \frac{L_{\mathrm{Pl}}}{R}
$$

where $L_{\mathrm{Pl}}=(\hbar G)^{\frac{1}{2}}$ is the Planck length (Emelyanov 2016a). In the leading-order approximation $T_{L}$ does not directly depend on the mass $M$ of the black hole. The quantum vacuum unlike a physical hot plasma described by $\alpha$ and $T$ (but not, e.g., by a particle density $n$ ) is effectively also characterised by $T_{L}$ which unlike $T_{H}$ plays a major role in local physics.

The wavelength of the mode has to be much smaller than the distance to the black hole. Moreover, it must be much smaller than a detector size $l_{D}$. This implies $m_{e}^{2} / e T_{H} \gg|\mathbf{k}|$ and $|\mathbf{k}| \gg 1 / l_{D} \gg 10^{2} \omega_{\mathrm{p}}$, where $\omega_{\mathrm{p}} \equiv \frac{1}{3} e T_{L}$ is a plasma-like frequency (Emelyanov 2016a) and the upper threshold is to suppress higher loop corrections (Emelyanov 2016c \& 2016a). In this case, plasmons are not propagating modes (Le Bellac 1996), whereas photons acquire an effective mass

$$
m_{\gamma} \approx \frac{1}{\sqrt{6}} e T_{L} \approx 2.5 \times 10^{-9}\left(\frac{1 \mathrm{~m}}{R}\right) \mathrm{eV} .
$$

In the physical plasma, plasmons are quasi-particles, i.e. collective excitations mediated by plasma particles. In our case, the vacuum is full of virtual particles, rather than real ones. Thus, the result of having no plasmons seems to be physically sensible.

A static electric field $\mathbf{E}$ sourced by a point-like charge $q$ is screened in the physical plasma - a phenomenon known as the Debye screening. This effect is due to a collective response of the plasma particles on putting the charge $q$ in the system. The linear response theory in our case yields a similar result, namely $\mathbf{E}=-\nabla \varphi$ with the electromagnetic potential $\varphi=(q / r) e^{-r / r_{L}}$, where

$$
r_{L}=\left(\sqrt{2} m_{\gamma}\right)^{-1} \approx 3.5 \times 10^{2} R
$$


Thus, the Debye-like length $r_{L}$ is much bigger than $R$. It means the screening is a tiny effect, because $l_{D} \ll R$ should hold for our approximation be reliable (at distances $\left|r-r^{\prime}\right| \gtrsim R$ the propagator (2.3) is invalid). With the technology of Williams et al. (1971) this can in principle be tested if such small black holes do exist in nature and one of them is within $R \lesssim 250 \mathrm{~km}$ from the detector. For a practical purpose, however, the probability of having such a black hole per cubic centimeter should be estimated to decide whether it is realistic to probe this effect during a reasonable amount of time.

If the black-hole evaporation lasts till its complete disappearance, then we should set a lower bound on the black-hole mass $M$ to guarantee the quasi-equilibrium approximation we have exploited above. It would be of order of $10^{10} \mathrm{~g}$ corresponding to the black-hole lifetime of one day. The initial mass of this primordial hole (i.e. $10^{10}$ years ago) should be in the range $3 \times 10^{14} \mathrm{~g} \lesssim M_{0} \ll 10^{16} \mathrm{~g}$, which corresponds to $10^{10} \mathrm{~g} \lesssim M \ll 10^{16} \mathrm{~g}$ today.

\section{Discussions}

We have considered three examples when the quantum vacuum can be envisaged as possessing medium-like properties. The virtual particles were understood as constituents of the vacuum like the real particles of ordinary media - the point of view which seems to be widely-accepted by the researchers.

As with any analogy, this viewpoint cannot be taken literally. Indeed, the real particles can transfer energy, whereas the virtual particles seem not be capable of doing that, because these are just fluctuations, although quantum in nature.

To clarify this aspect of the quantum fluctuations, one may put an extra conducting plate between the Casimir plates. It may be taken to be freely moving and at the distance $l_{C} / 2$ from each plates at $t<0$. Its motion is however fixed by $v(t)=v \theta(t) \theta(t-\tau) \ll 1$. It follows from energy conservation that a part of the vacuum energy in both cavities will by absorbed by the middle plate during its motion. In other words, the vacuum energy is partially dissipated in the middle plate and partially redistributed between the cavities. The dissipative part could result in heating of the middle plate with an increase in temperature of $\Delta T \propto V(v \tau)^{2} / l_{C}^{6}$ in case $v \tau \ll l_{C}$, where $V$ is its volume. This gedankenexperiment might imply the virtual particles are capable of transferring energy.

\section{Acknowledgements}

I am grateful to the organisers of IAU Symposium 324 for the financial support. It is a pleasure to thank the anonymous referee for his physically relevant comments/questions.

\section{References}

Bittencourt, J. A., 2005, Fundamentals of Plasma Physics, Springer-Verlag

Dittrich, W. \& Gies, H., 2000, Probing the quantum vacuum. Perturbative effective action approach in Quantum Electrodynamics and its application, Springer-Verlag

Drummond, I. T. \& Hathrell, S. J., 1980, Phys. Rev. D, 22, 343

Emelyanov, S., 2016a, arXiv:1603.01148

Emelyanov, S., 2016b, arXiv:1608.05318

Emelyanov, S., 2016c, Nucl. Phys. B, 913, 318

Haag, R., 1996, Local Quantum Physics. Fields, Particles, Algebras, Springer-Verlag

Le Bellac, M., 1996, Thermal field theory, Cambridge University Press

Melrose, D. B. \& Stoneham, R. J., 1976, Nuovo Cim. A, 32, 435

Mukhanov, V. F. \& Chibisov, G. V., 1981, JETP Lett., 33, 532

Peskin, M. E. \& Schroeder, D. V., 1995, An Introduction to Quantum Field Theory, AddisonWesley Publishing

Weldon, H. A., 1982, Phys. Rev. D, 26, 1394

Williams, E. R., Faller, J. E., \& Hill, H. A., 1971, Phys. Rev. Lett. 26, 721 